\title{
Efeito de um Resíduo do Processo de Galvanoplastia sobre a Vulcanização da Borracha Natural (NR)
}

\author{
Alex C. S. Santos, Helson M. da Costa, Valéria D. Ramos \\ Departamento de Materiais, Instituto Politécnico, UERJ
}

\begin{abstract}
Resumo: Um resíduo gerado no processo de galvanoplastia (RG), rico em metais, foi incorporado em composições de borracha natural (NR) em substituição parcial ou total ao ativador comum do processo de vulcanização, o óxido de zinco $(\mathrm{ZnO})$. As diferentes misturas foram preparadas em um misturador de calandras e, após a reometria, foram vulcanizadas a $150{ }^{\circ} \mathrm{C}$. As propriedades mecânicas de resistência à tração e rasgo foram determinadas, além do teor de gel. Além disso, o efeito do resíduo RG sobre a cinética de vulcanização foi analisado através do modelo de Coran, usando-se as temperaturas de 150,160 e $170{ }^{\circ} \mathrm{C}$. Os resultados mostraram que RG desenvolve algum efeito catalítico sobre a vulcanização de NR, embora exista uma considerável perda na resistência à tração quando o teor de RG excede o do ZnO nas composições. Quanto ao rasgo, a propriedade é mantida em um patamar satisfatório enquanto teores menores de RG são utilizados.
\end{abstract}

Palavras-chave: Borracha natural, resíduo de galvanoplastia, vulcanização, propriedades mecânicas.

\section{Effect from the Waste from the Electroplating Process on the Natural Rubber (NR) Vulcanization}

\begin{abstract}
A waste generated in the electroplating process (RG), rich in metals, was incorporated in natural rubber (NR) compositions, as a partial or complete replacement of zinc oxide $(\mathrm{ZnO})$, which is a typical activator of the vulcanization process. Different mixtures were prepared in a two-roll mixer, which were vulcanized at $150{ }^{\circ} \mathrm{C}$ after the rheometry measurements. Mechanical properties such as tensile and tear strength were evaluated, as well as the gel content. In addition, the effect of RG waste upon the vulcanization kinetics was analyzed with the Coran model using temperatures at 150 , 160 and $170{ }^{\circ} \mathrm{C}$. The results showed that RG develops some catalytic effect upon NR vulcanization, but there is a poor mechanical performance when $\mathrm{RG}$ loading is higher than that of $\mathrm{ZnO}$ in the compositions. For the tear strength, the property is maintained at a reasonable level if a small RG loading is utilized.
\end{abstract}

Keywords: Natural rubber, electroplating waste, vulcanization, mechanical properties.

\section{Introdução}

A partir da Revolução Industrial o homem moderno passou a satisfazer suas necessidades quase que plenamente calcado no modelo urbano-industrial de desenvolvimento. Muito embora as atividades industriais fabriquem materiais de necessidade, também produzem resíduos diversos em quantidade e diversidade, os quais são sempre causadores de pelo menos um tipo de poluição ou contaminação.

A estrutura produtiva do segmento Metal Mecânico em Nova Friburgo está baseada nas micros, pequenas e médias empresas atuando para o mercado da construção civil e automobilístico, garantindo grande papel empregatício na região. As empresas da atividade metal mecânica podem ser classificadas como fabricantes de artefatos de metal e de peças fundidas, essencialmente de metais não ferrosos e suas ligas, e em muitas a galvanoplastia é a principal forma de acabamento e embelezamento.

A galvanoplastia é o processo no qual certos materiais, principalmente metálicos, sofrem revestimento para adquirirem uma proteção contras às intempéries e ao manuseio, além de conferência de beleza, durabilidade e melhoria das propriedades superficiais para satisfazer às necessidades e exigências do mercado. Em geral, os resíduos da indústria galvânica são enquadrados na Classe I devido às suas propriedades físico-químicas ou infecto-contagiosas. Tais resíduos quando manuseados e gerenciados de forma indevida podem acarretar danos à sanidade ambiental e pública. As formas de poluição e contaminação da galvanoplastia podem ser muitas desde emissões gasosas, resíduos sólidos e efluentes líquidos, até a estação de tratamento de efluentes industriais na qual é gerado o lodo galvânico, o qual é considerado como resíduo Classe $I^{[1-3]}$.

O ZAMAC, liga de zinco com alumínio, magnésio e cobre, é o material mais utilizado na galvanoplastia devido às propriedades mecânicas e físicas de tração, resistência à corrosão, ao desgaste e choques, além das características de fundição e de eletrodeposição. As imperfeições do ZAMAC como impurezas, falhas, riscos, zonas requentadas, manchas,

Autor para correspondência: Helson M. da Costa, Departamento de Materiais, Instituto Politécnico, Universidade do Estado do Rio de Janeiro, 
poros e lacunas contribuem para a diminuição da vida útil da peça. A fim de que exista resistência, é preciso efetuar alguns processos físico-químicos de tratamento de superfície da peça a ser formada (jateamento, esmerilhamento, polimento, tamboreamento e vibração, desengraxe, lavagem, decapagem e neutralização) com intuito de que haja a deposição uniforme e necessária sobre o material a ser revestido ${ }^{[1-3]}$.

Em paralelo, no processo de vulcanização de elastômeros de uso geral como, por exemplo, a borracha natural (NR), o copolímero estireno-butadieno (SBR), a borracha nitrílica (NBR), a borracha butílica (IIR), entre outros, o óxido de zinco $(\mathrm{ZnO})$ é um ingrediente costumeiro das formulações, sendo incorporado às borrachas em teores de $2 \mathrm{a} 10 \mathrm{~g}$ por $100 \mathrm{~g}$ de borracha ( 2 a $10 \mathrm{phr}$ ). $\mathrm{ZnO}$, juntamente com um ácido graxo (geralmente, ácido esteárico), desenvolve o papel de ativador do sistema de vulcanização. $\mathrm{O}$ ácido graxo e o $\mathrm{ZnO}$ formam um sal que, posteriormente, pode formar complexos com os aceleradores orgânicos e, desta forma, promover uma satisfatória rede de ligações cruzadas na borracha ${ }^{[4]}$.

Nesta investigação, o resíduo do processo de polimento das peças metálicas de ZAMAC, denominado RG, foi avaliado como um possível ativador para um sistema de vulcanização convencional $(\mathrm{CV})$ aplicado à borracha natural (NR). Tal resíduo possui em sua composição química uma riqueza em metais, o que pode torná-lo potencialmente útil na substituição do óxido de zinco, ativador costumeiro dos sistemas CV de borrachas de uso geral.

\section{Experimental}

Todos os materiais foram usados como recebidos. Borracha natural foi fornecida pela Michelin Brasil. O resíduo do processo de galvanoplastia (RG) foi obtido junto à empresa Stam Metalúrgica S.A. O antioxidante usado, Aminox, foi doado pela Uniroyal Química S.A. O acelerador $\mathrm{N}$-t-butil-2-benzotiazol-sulfenamida (TBBS) foi doado pela Bann Química. Outros ingredientes da composição, tais como, óxido de zinco, ácido esteárico e enxofre foram reagentes de grau analítico.

RG foi moído em moinho de bolas por 5 horas e peneirado em malha de 325 mesh. Após calcinação em $550{ }^{\circ} \mathrm{C}$ por um período de 4 horas, a composição química do resíduo (Tabela 1) foi determinada por Espectrometria de Emissão Atômica por Plasma Acoplado Indutivamente. A formulação usada está na Tabela 2, a mesma foi utilizada para no preparo das composições de borracha natural contendo diferentes razões de $\mathrm{ZnO} / \mathrm{RG}$.

As misturas foram conduzidas em um misturador de rolos usando-se uma razão de fricção de $1: 1,25$ e $70^{\circ} \mathrm{C}$, de acordo com a norma ASTM D 3182. Cinco composições foram preparadas e o curômetro TI-100, Tecnología Industrial, foi empregado com arco de $\pm 1^{\circ}$ para a obtenção das curvas de torque-tempo. Três temperaturas foram selecionadas para a vulcanização 150,160 e $170{ }^{\circ} \mathrm{C}$. Para cada temperatura, duas amostras de cada mistura foram analisadas. Os dados
Tabela 1. Composição química do resíduo do processo de galvanoplastia (RG).

\begin{tabular}{ccc}
\hline $\begin{array}{c}\text { Elemento } \\
\text { químico }\end{array}$ & $\begin{array}{c}\text { Valor de } \\
\text { eletronegatividade }\end{array}$ & $\begin{array}{c}\text { Concentração } \\
\text { (ppm) }\end{array}$ \\
\hline $\mathrm{Al}$ & 1,61 & 691 \\
$\mathrm{Cd}$ & 1,69 & $<0,20$ \\
$\mathrm{Ca}$ & 1,00 & 212 \\
$\mathrm{~Pb}$ & 1,80 & 3,8 \\
$\mathrm{Cu}$ & 1,90 & 1.109 \\
$\mathrm{Cr}$ & 1,66 & 3,9 \\
$\mathrm{Fe}$ & 1,83 & 190 \\
$\mathrm{P}$ & 2,19 & 484 \\
$\mathrm{Mg}$ & 1,31 & 675 \\
$\mathrm{Mn}$ & 1,55 & 58 \\
$\mathrm{Ni}$ & 1,91 & 28 \\
$\mathrm{Zn}$ & 1,65 & 29.190 \\
$\mathrm{Si} \mathrm{e} \mathrm{insolúveis}$ & 1,90 & 517.000 \\
\hline & & \\
\hline
\end{tabular}

Tabela 2. Composição das diferentes misturas de borracha natural contendo o resíduo $\mathrm{RG}$.

\begin{tabular}{lcc}
\hline \multicolumn{1}{c}{ Material } & \multicolumn{2}{c}{ Concentração (phr) } \\
\hline Borracha natural (NR) & \multicolumn{2}{c}{100} \\
Ácido esteárico & \multicolumn{2}{c}{2,5} \\
TBBS $^{\mathrm{a}}$ & \multicolumn{2}{c}{0,8} \\
Aminox $^{\mathrm{b}}$ & \multicolumn{2}{c}{2,0} \\
Enxofre & \multicolumn{2}{c}{2,5} \\
ZnO/RG & $\mathrm{M}_{1}$ & $4,0 / 0,0$ \\
& $\mathrm{M}_{2}$ & $3,0 / 1,0$ \\
& $\mathrm{M}_{3}$ & $2,0 / 2,0$ \\
& $\mathrm{M}_{4}$ & $1,0 / 3,0$ \\
& $\mathrm{M}_{5}$ & $0,0 / 4,0$ \\
\hline
\end{tabular}

${ }^{\mathrm{a} N} \mathrm{~N}$ - -butil-2-benzotiazol-sulfenamida; ${ }^{\mathrm{b} A n t i o x i d a n t e: ~ p r o d u t o ~ d a ~}$ reação em baixa temperatura entre acetona e difenilamina.

reométricos foram usados para o cálculo dos parâmetros cinéticos - velocidade de reação e energia de vulcanização.

Para as propriedades mecânicas, os vulcanizados foram preparados por moldagem por compressão a $150{ }^{\circ} \mathrm{C}$ e 3,0 $\mathrm{MPa}$ em uma prensa hidráulica aquecida eletricamente. Após o corte dos corpos de prova e o condicionamento por 24 horas, as propriedades de resistência à tração (ASTM D 412) e rasgo (ASTM D 624) foram determinadas em uma máquina de ensaios universal Shimadzu, Model AG-I, com célula de $1 \mathrm{kN}$. O teor de gel para as diferentes composições de borracha natural foi estimado usando-se xileno como solvente (ASTM D 2765).

\section{Resultados e Discussão}

\section{Propriedades mecânicas e reológicas}

A Tabela 3 apresenta os dados reométricos obtidos para as diferentes composições de NR em $150{ }^{\circ} \mathrm{C}$. O tempo de précura (scorch time) define o tempo necessário para o início da vulcanização em uma dada temperatura e, portanto, representa 
o tempo disponível para o processamento. O tempo ótimo de cura é o tempo necessário durante a etapa de vulcanização, em uma dada temperatura, para que uma quantidade suficiente de ligações cruzadas seja formada, o que conduz a um nível desejado em termos de propriedade ${ }^{[5]}$.

Pode ser verificado que a adição contínua de RG, ou seja, misturas que contêm proporções cada vez maiores de RG em relação ao $\mathrm{ZnO}$, apresentam uma sutil redução nos valores no tempo de pré-cura $\mathrm{t}_{\mathrm{s} 1}$ (aproximadamente $8,5 \%$ de $\mathrm{M} 1$ para M5) e tempo ótimo de vulcanização $t_{90}$ (em torno de 10,8\% de M1 para M5). Embora a presença do resíduo galvânico possa introduzir algum efeito catalítico sobre a vulcanização de NR, isso não implicou na formação de redes de ligações cruzadas satisfatórias. Tal observação é fundamentada na progressiva redução dos valores de torque máximo e teor registrados para as diferentes composições. Há uma diminuição de $58 \%$ em $\mathrm{M}_{\mathrm{h}}$ da mistura M1 para a mistura M5.

Na Tabela 4, os dados das propriedades mecânicas e teor de gel são apresentados. Os valores encontrados corroboram com a redução vista em $\mathrm{M}_{\mathrm{h}}$. Os vulcanizados que contêm maior proporção de RG mostram pequeno teor de gel e baixo desempenho mecânico, sobretudo quanto à resistência à tração e a tenacidade (capacidade do material absorver energia até a fratura). A mistura M5, por exemplo, realizada apenas com RG como ativador, produz vulcanizados com resistência à tração $82,4 \%$ inferior aos vulcanizados oriundos da mistura M1, apenas com $\mathrm{ZnO}$. Apenas na propriedade de rasgo, há uma certa equivalência de desempenho.

Baseado em trabalhos anteriores, realizados com cinza da casca de arroz, é sugerido que a presença de elementos metálicos na composição química do resíduo de galvanoplastia (RG) possa ter trazido um efeito sinérgico e, desta forma, ter tornado a vulcanização mais rápida ${ }^{[6-8]}$. Russel et al ${ }^{[9]}$, em investigação do efeito de óxidos $\left(\mathrm{MgO}, \mathrm{SnO}_{2}, \mathrm{~Pb}_{3} \mathrm{O}_{4}, \mathrm{Sb}_{2} \mathrm{O}_{3}\right.$, $\mathrm{TeO}_{2}, \mathrm{Fe}_{2} \mathrm{O}_{3}, \mathrm{Cr}_{2} \mathrm{O}_{3} \mathrm{e} \mathrm{Co}_{2} \mathrm{O}_{3}$ ) sobre um sistema de vulcanização eficiente, demonstraram que embora nenhum outro óxido seja tão eficiente quanto o óxido de zinco, alguns podem mostrar

Tabela 3. Dados reométricos a $150{ }^{\circ} \mathrm{C}$ das misturas de NR contendo diferentes razões $\mathrm{ZnO/RG}$.

\begin{tabular}{cccc}
\hline Mistura & $\begin{array}{c}\text { Tempo de pré- } \\
\text { cura }\left(\mathbf{t}_{\mathbf{s} \mathbf{1}}, \mathbf{m i n}\right)\end{array}$ & $\begin{array}{c}\text { Tempo ótimo de } \\
\text { cura }\left(\mathbf{t}_{\mathbf{9}}, \mathbf{m i n}\right)\end{array}$ & $\begin{array}{c}\text { Torque máximo } \\
(\mathbf{M h}, \mathbf{d N} \cdot \mathbf{m})\end{array}$ \\
\hline $\mathrm{M}_{1}$ & $7,8 \pm 0,1$ & $13,0 \pm 0,2$ & $21,5 \pm 0,2$ \\
$\mathrm{M}_{2}$ & $7,6 \pm 0,2$ & $13,9 \pm 0,4$ & $21,9 \pm 0,2$ \\
$\mathrm{M}_{3}$ & $7,5 \pm 0,4$ & $12,6 \pm 1,1$ & $19,8 \pm 2,6$ \\
$\mathrm{M}_{4}$ & $7,1 \pm 0,4$ & $11,1 \pm 0,3$ & $17,2 \pm 0,3$ \\
$\mathrm{M}_{5}$ & $6,5 \pm 0,1$ & $11,6 \pm 2,0$ & $9,0 \pm 0,2$ \\
\hline
\end{tabular}

um pequeno incremento de desempenho em conjunto com $\mathrm{ZnO}$. Por outro lado, para entender porque o óxido de zinco é preferido entre os óxidos metálicos, um estudo comparativo foi conduzido com os óxidos de titânio, magnésio, cálcio, chumbo e zinco ${ }^{[10]}$. Foi observado através da correlação entre a eletronegatividade $v s$. incremento no valor de torque registrado $\left(\Delta \mathrm{M}=\mathrm{M}_{\mathrm{h}}-\mathrm{M}_{\mathrm{l}}\right.$, onde $\mathrm{M}_{1}$ é o torque mínimo $)$ que boas propriedades nos vulcanizados são alcançadas somente quando uma faixa ótima de eletronegatividade é obedecida $(1,6$ a 1,8$)$.

Na Tabela 1, observa-se que os componentes metálicos presentes em quantidades mais significativas na composição de RG estão fora da faixa recomendada de eletronegatividade. Assim sendo, a estabilidade eletrônica do complexo formado pelo acelerador e/ou seus produtos de reação com enxofre e o elemento metálico M qualquer é comprometida. Desta forma, com um número reduzido da espécie química em questão, o processo de vulcanização não ocorre em extensão suficiente e, conseqüentemente, há redução de desempenho mecânico para os vulcanizados contendo RG em proporção dominante.

Além disso, a borracha natural, assim como muitos outros polímeros, é altamente suscetível à degradação devido à presença de ligações duplas na cadeia principal. A degradação de NR é acelerada principalmente pelo calor, umidade, luz, ozônio, radiação etc. Vinod et al ${ }^{\left[{ }^{[1]}\right.}$ em estudo sobre a incorporação de alumínio e cargas comercias (negro de fumo e sílica) em composições de NR, demonstra que cargas dispersas influenciam consideravelmente não apenas as propriedades dos compósitos, mas também sua degradação e estabilidade. Lokander et al. ${ }^{[12]}$ observaram que a estabilidade oxidativa da NR decresce pronunciadamente em presença de grande quantidade de partículas de ferro na composição.

Assim sendo, a presença de alguns elementos metálicos, em especial o cobre $(\mathrm{Cu})$, na composição do resíduo $\mathrm{RG}$ pode também ter contribuído para a degradação oxidativa com a conseqüente redução do número de ligação cruzadas e perda de desempenho mecânico em composições onde o resíduo é utilizado em maior proporção. A fim de melhor avaliar o efeito de RG sobre a vulcanização, um estudo cinético foi conduzido usando-se o modelo proposto por Coran ${ }^{[13]}$.

\section{Cinética de vulcanização}

Para o estudo da cinética de vulcanização das misturas de NR contendo diferentes razões de $\mathrm{ZnO} / \mathrm{RG}$, o modelo de Coran $^{[13]}$ foi empregado. O modelo foi desenvolvido a

Tabela 4. Propriedades mecânicas para as composições de NR contendo diferentes razões ZnO/RG.

\begin{tabular}{ccccccc}
\hline Mistura & $\begin{array}{c}\text { Módulo elástico à } \\
\mathbf{1 0 0 \%}(\mathbf{k P a})\end{array}$ & $\begin{array}{c}\text { Resistência à } \\
\text { tração }(\mathbf{M P a})\end{array}$ & $\begin{array}{c}\text { Alongamento na } \\
\text { ruptura }(\%)\end{array}$ & $\begin{array}{c}\text { Energia para a } \\
\text { ruptura }(\mathbf{J})\end{array}$ & $\begin{array}{c}\text { Rasgo } \\
\left(\mathbf{N} / \mathbf{m m}^{2}\right)\end{array}$ & $\begin{array}{c}\text { Teor de } \\
\text { gel }(\boldsymbol{\%})\end{array}$ \\
\hline $\mathrm{M}_{1}$ & $485,5 \pm 43,7$ & $18,8 \pm 0,9$ & $1200 \pm 35$ & $41,1 \pm 5,6$ & $2,2 \pm 0,2$ & $85 \pm 1$ \\
$\mathrm{M}_{2}$ & $426,2 \pm 35,7$ & $13,5 \pm 1,5$ & $1045 \pm 40$ & $38,9 \pm 3,3$ & $2,3 \pm 0,1$ & $61 \pm 2$ \\
$\mathrm{M}_{3}$ & $414,3 \pm 20,5$ & $10,6 \pm 2,0$ & $980 \pm 45$ & $34,3 \pm 2,5$ & $2,3 \pm 0,3$ & $45 \pm 2$ \\
$\mathrm{M}_{4}$ & $296,8 \pm 40,0$ & $7,6 \pm 0,2$ & $900 \pm 36$ & $28,4 \pm 2,6$ & $1,7 \pm 0,2$ & $25 \pm 1$ \\
$\mathrm{M}_{5}$ & $178,9 \pm 38,8$ & $3,3 \pm 0,8$ & $840 \pm 44$ & $3,5 \pm 0,4$ & $0,8 \pm 0,2$ & $3 \pm 1$ \\
\hline
\end{tabular}


partir dos dados experimentais, obtidos por análise química, de vulcanizados de NR com enxofre e 2-(morfolinotio) benzotiazol como acelerador ${ }^{[13,14]}$.

A sequiência de reações do modelo é apresentada no Esquema 1 e na Figura 1, onde A é o acelerador e/ou seus produtos de reação (com enxofre, $\mathrm{Zn}^{2+}$, etc.); $\mathrm{B}$ é um precursor de ligações cruzadas, provavelmente polimérico; $\mathrm{B}^{*}$ é a forma ativada de B, provavelmente um radical livre; Vu é a ligação cruzada; $\alpha$ e $\beta$ são ajustes estequiométricos. A borracha não é usada no esquema porque é considerada estar em excesso
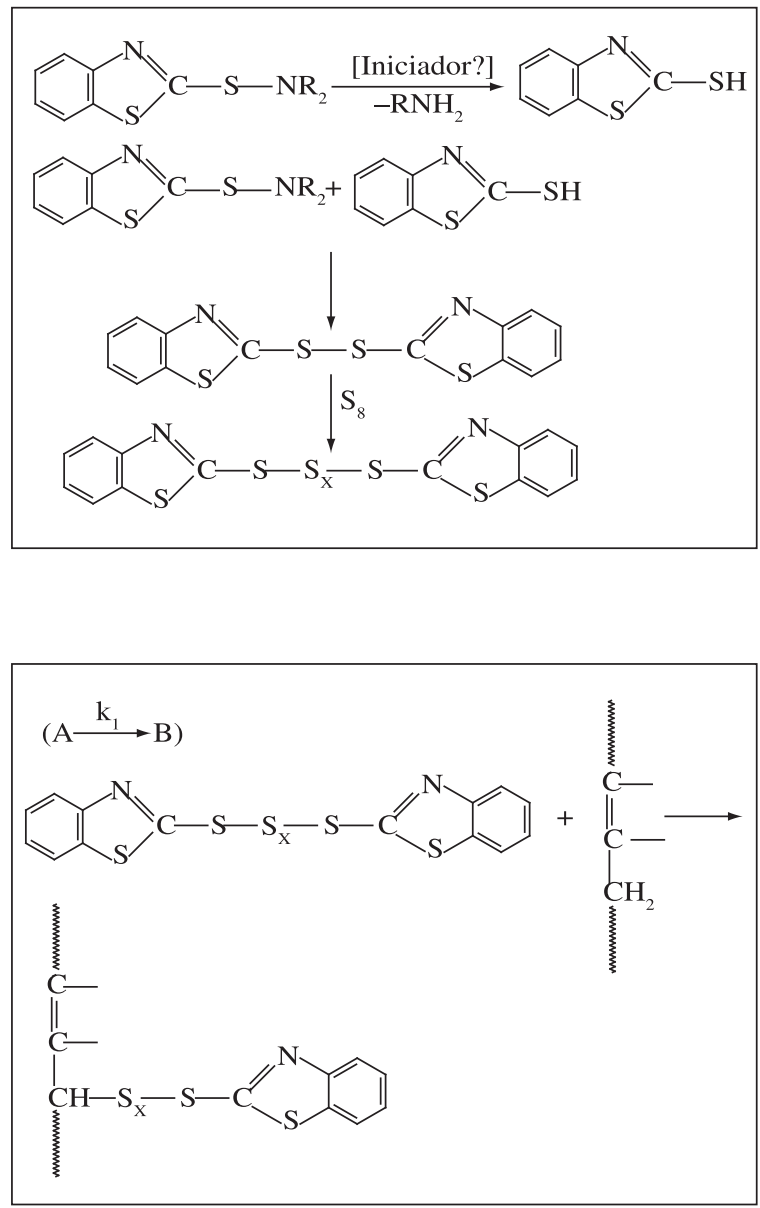

e, portanto, com uma concentração essencialmente constante em todo o processo. O modelo explica os longos tempos de pré-cura encontrados em vulcanizações conduzidas com aceleradores do tipo tiazóis sulfenamidas; se a reação através de $\mathrm{k}_{4}$ é muito mais rápida do que aquela através de $\mathrm{k}_{3}$, muito pouca formação de ligação de cruzada pode ocorrer até que a espécie A seja essencialmente consumida. Ambas as reações através de $\mathrm{k}_{3}$ e $\mathrm{k}_{4}$ são consideradas serem muito mais rápidas do que a reação através de $\mathrm{k}_{2}$. Portanto, a etapa controladora
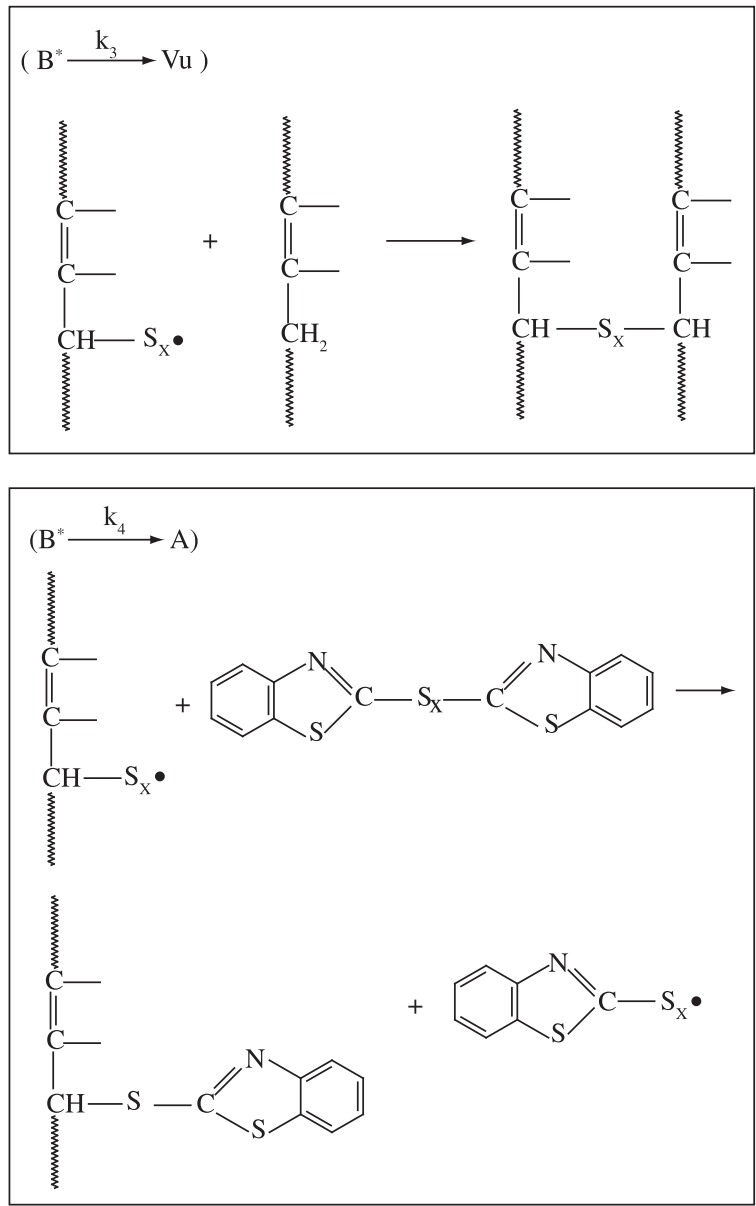

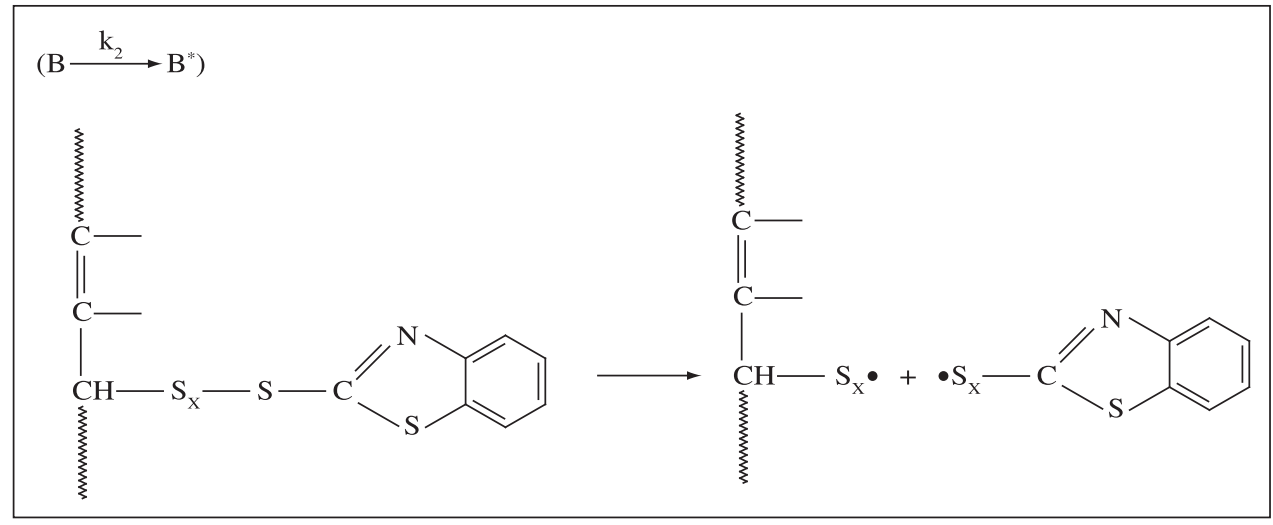

Figura 1. Modelo de Coran ${ }^{[10,11]}$ para vulcanização acelerada com sulfenamidas e formação de A, B, B* e Vu, e reações laterais. 
da velocidade da reação, após o completo consumo de A, para a formação de ligações cruzadas é definida por $\mathrm{k}_{2}^{[13,14]}$.

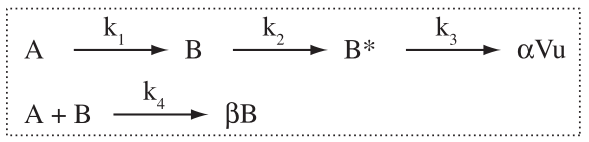

Esquema 1: Sequiência reacional simples para a cinética de vulcanização de composições com tempo de pré-cura significativo $^{[13,14]}$.

A constante de velocidade $\mathrm{k}_{2}$ pode ser determinada a partir de dados do reômetro de disco oscilatório assumindose que a formação de $\mathrm{Vu}$ é de primeira ordem com relação à espécie B. Assume-se também que o progresso da cura $\left(\mathrm{L}_{\mathrm{t}}\right.$, medido no reômetro), correspondente a qualquer período de vulcanização (t), é proporcional a concentração de Vu. Além disso, o aumento nas leituras de torque no reômetro $\left(\mathrm{L}_{\mathrm{t}}\right)$ são proporcionais à densidade de ligações cruzadas, embora a constante de proporcionalidade entre $\mathrm{L}_{\mathrm{t}}$ e $\mathrm{V}_{\mathrm{ut}}$ mude de acordo com o tipo de composição ${ }^{[14]}$. A seguinte equação proposta por Cotten ${ }^{[15]}$ pode ser usada para a estimativa de $\mathrm{k}_{2}$ :

$$
\ln \left[1-\left(\frac{\Delta \mathrm{L}_{\mathrm{t}}}{\Delta \mathrm{L}}\right)\right]=\mathrm{k}_{2} \mathrm{t}
$$

onde $\mathrm{L}$ representa o aumento máximo no valor do torque do reômetro observado para uma vulcanização completa ou após t minutos; e, $\mathrm{k}_{2}$ é a constante de velocidade para a reação de ligação cruzada. A natureza de primeira ordem não é alcançada imediatamente até que o início da formação de ligações cruzadas ocorra de fato. O tempo necessário para que a curvatura desapareça no gráfico gerado pela Equação 1, o qual é o tempo requerido para a reação tornar-se de primeira ordem, é denominado como tempo de tdisplay, ou seja, tempo preciso para o completo consumo de A. Após o tdisplay, o gráfico da Equação 1 gera uma reta e $\mathrm{k}_{2}$ é, então, a inclinação negativa da porção linear da curva ${ }^{[13-15]}$. Para curvas de reômetro, os valores de $k_{1}$ e $k_{2}$ podem ser relacionados por meio de um gráfico logarítmico e a solução da Equação 2 abaixo:

$$
\mathrm{k}_{1} * \mathrm{t}_{\text {display }}-\ln \mathrm{k}_{1}=\mathrm{k}_{2} * \mathrm{t}_{\text {display }}-\ln \mathrm{k}_{2}
$$

Nas Figuras 2 e 3, as constantes de velocidade $\mathrm{k}_{2}$ e $\mathrm{k}_{1}$ do modelo de Coran ${ }^{[13,14]}$ foram medidas em três diferentes temperaturas para que o cálculo da energia de ativação fosse possível. Em ambos os casos, o efeito global do aumento da temperatura é verificado, ou seja, a cada $10^{\circ} \mathrm{C}$ de incremento há, aproximadamente, uma duplicação das constantes de velocidade.

Além disso, verifica-se que o efeito da adição contínua do resíduo RG é mais pronunciado sobre a etapa de geração de B, um precursor de ligações cruzadas (Figura 3). O valor médio registrado para $\mathrm{k}_{1}$ na composição $\mathrm{M} 5$, apenas com RG como ativador, é cerca de 3,6 maior do que aquele verificado na composição $\mathrm{M} 1$, apenas com $\mathrm{ZnO}$. Isto significa uma

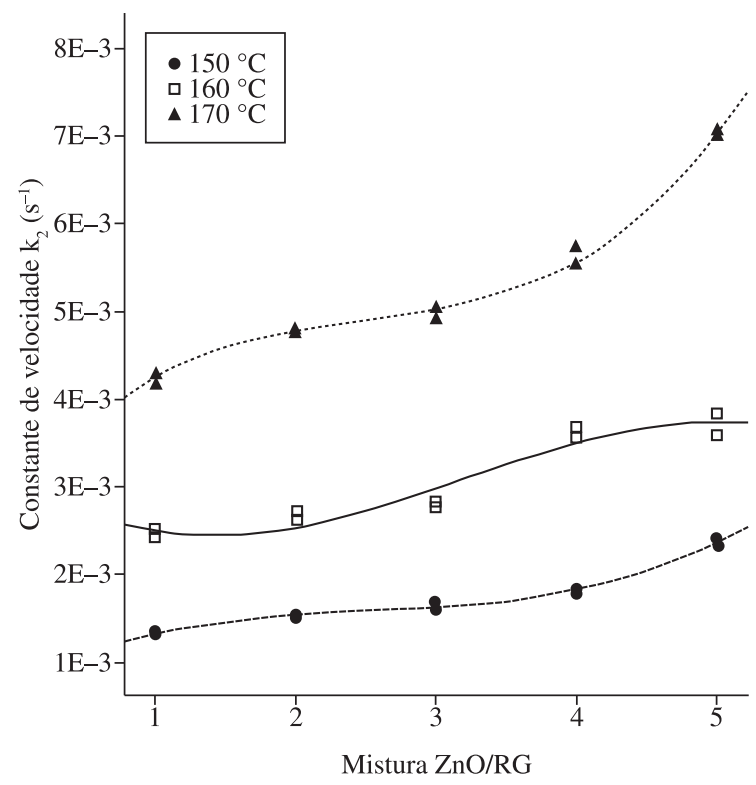

Figura 2. Constante de velocidade $\mathrm{k}_{2}$ para as composições de NR contendo diferentes razões $\mathrm{ZnO} / \mathrm{RG}$.

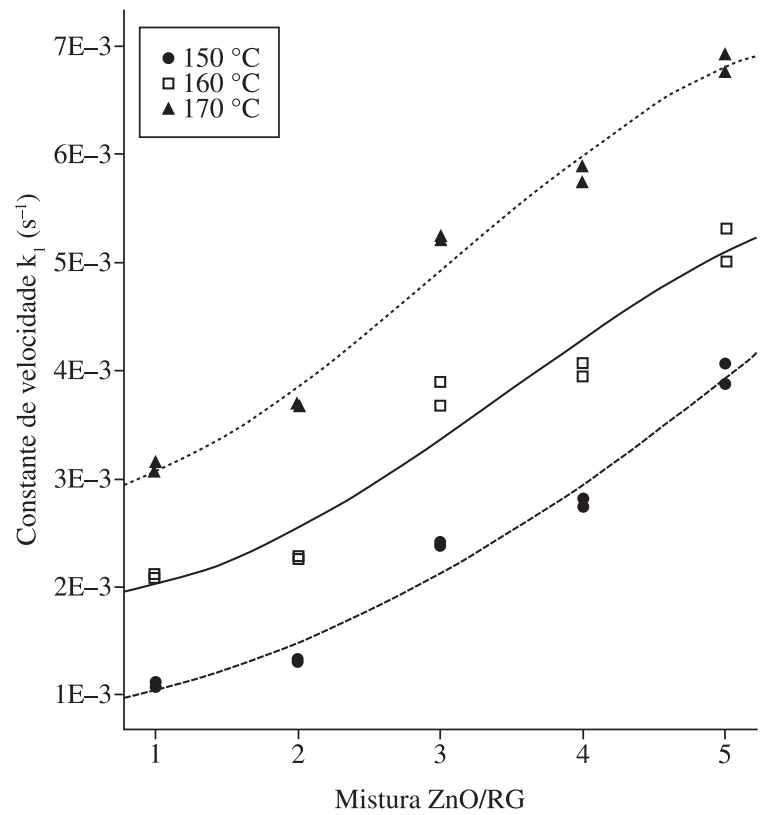

Figura 3. Constante de velocidade $\mathrm{k}_{1}$ para as composições de NR contendo diferentes razões $\mathrm{ZnO} / \mathrm{RG}$.

formação mais rápida da espécie $\mathrm{A}$ (acelerador e/ou seus produtos de reação com enxofre, $\mathrm{Zn}^{2+}$, etc.), o que corrobora $\mathrm{o}$ aumento de $\mathrm{k}_{1}$ e a diminuição verificada nos tempos de précura $\left(t_{\mathrm{s} 1}\right)$. $\mathrm{O}$ efeito sinérgico entre os diferentes componentes metálicos do resíduo RG e o óxido de zinco durante a vulcanização pode ser sugerido para a validação dos dados experimentais. O cálculo da energia de ativação da etapa correspondente a $k_{1}$ também foi realizado e na Figura 4 verifica-se que há um grande decréscimo de, mais ou menos, 48\% no valor médio da composição M1 para M5. Tal fato apenas reflete o já comentado efeito catalítico do resíduo RG nas composições de NR. 


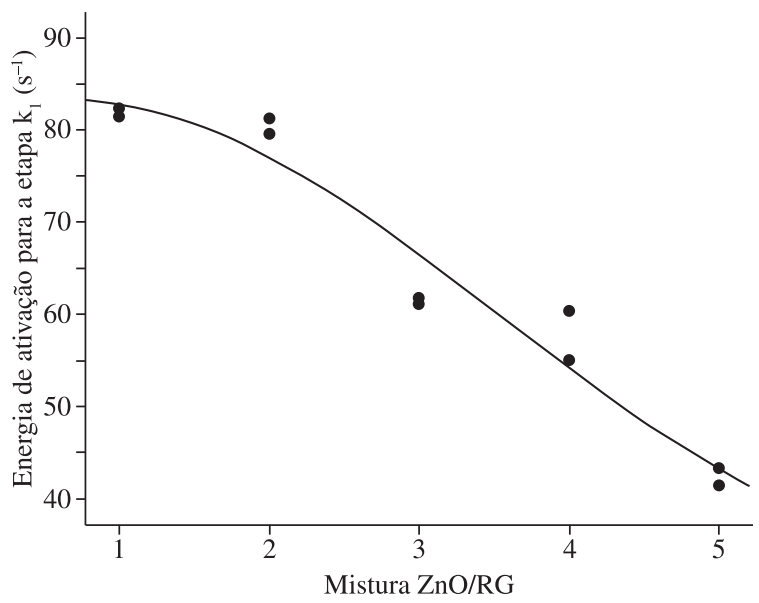

Figura 4. Energia de ativação para a etapa $k_{1}$ para as composições de NR contendo diferentes razões $\mathrm{ZnO} / \mathrm{RG}$.

Embora RG gere um efeito catalítico sobre a vulcanização, este efeito não se traduz em uma melhoria das propriedades dos vulcanizados conforme descrito no item (Propriedades mecânicas e reológicas). A adição de RG aumenta de forma sutil o valor da constante $\mathrm{k}_{2}$ (Figura 2) e reduz o valor da energia de ativação (Figura 5) em apenas 6,8\% quando comparamos os valores médios registrados de M1 e M5. Esse comportamento mostra que a presença contínua de $\mathrm{RG}$ em relação ao $\mathrm{ZnO}$ não apenas pouco altera a cinética da principal etapa do mecanismo de vulcanização, ou seja, a formação da espécie B* (Figura 1), mas também sugere que não há a formação de uma quantidade significativa da espécie $\mathrm{B}^{*}$ (precursor de ligações cruzadas, provavelmente um radical livre).

Muitos diferentes mecanismos para a vulcanização com enxofre foram sugeridos ao longo dos anos. Os caminhos propostos freqüentemente envolvem diversas reações consecutivas e/ou competitivas e podem envolver numerosos intermediários. Com o grande número de reações competitivas e o grande número de intermediários, a identificação de uma estrutura como intermediário crítico parece ser uma tarefa incomensurável e inviável. No entanto, a importância da formação de complexos sulfurantes (por exemplo, a geração da espécie A da Figura 1) têm sido evidenciada através de estudos que nasceram na primeira metade do século XX (relações quantitativas estrutura-atividade ou QSAR quantitative structure-activity relationships ${ }^{[16]}$.

Versloot et al. ${ }^{[17]}$ estudaram o mecanismo da vulcanização de borrachas usando o 2,2-dimetil-2-buteno, um composto orgânico de baixa massa molecular, e uma mistura de vários óxidos metálicos. Usando aceleradores baseados em dissulfetos de tetra-alquiltiuram, eles verificaram que $\mathrm{Cd}$, $\mathrm{Cu}, \mathrm{Pb}$ ou Ni não são bons substitutos para o zinco quando da formação de complexos intermediários durante o processo de vulcanização. Steudel et al. ${ }^{[18]}$ estudaram a formação de complexos entre o acelerador dissulfeto de tetrametiltiuram (TMTD) e compostos de zinco através de métodos de química quântica. Foi provado efetivamente pela primeira vez que o

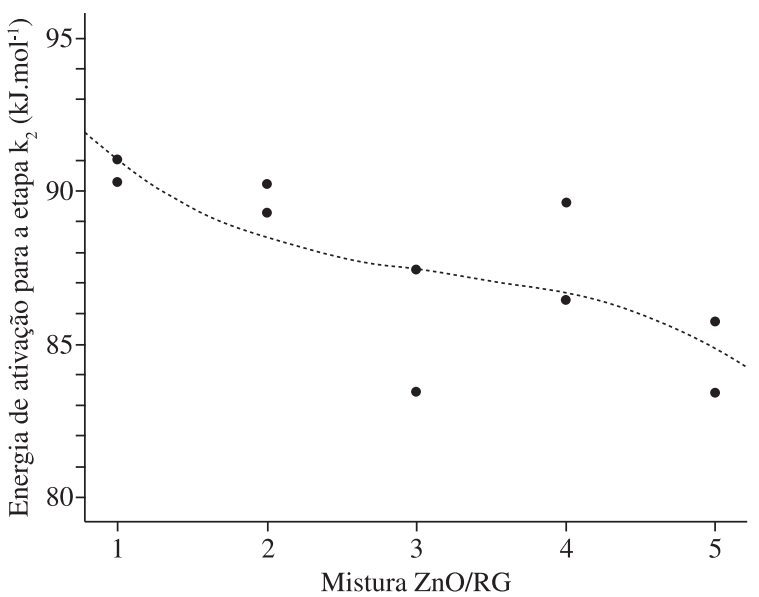

Figura 5. Energia de ativação para a etapa $\mathrm{k}_{2}$ para as composições de NR contendo diferentes razões $\mathrm{ZnO} / \mathrm{RG}$.

$\mathrm{ZnO}$ atua como ativador frente ao TMTD formando quelatos (compostos químicos formados por um íon metálico ligado por várias ligações covalentes a uma estrutura heterocíclica de compostos orgânicos) imprescindíveis para a vulcanização.

Assim sendo, a explicação para o efeito negativo de RG, conforme verificado no cálculo das constantes de velocidade do modelo de $\operatorname{Coran}^{[13,14]}$ e das energias de ativação, encontra suporte em trabalhos da literatura. Embora a presença crescente de RG nas composições de NR possa possibilitar a formação da espécie A mais rapidamente, o que é evidenciado, por exemplo, pela redução em $t_{\mathrm{s} 1}$, as espécies formadas ou não são em número suficiente ou não satisfazem os requisitos necessários para a formação de complexos sulfurantes realmente ativos ${ }^{[10,16-18]}$. Logo, as espécies A geradas não são capazes de dar prosseguimento a uma vulcanização satisfatória (formação de $\mathrm{B}^{*} \mathrm{e} \mathrm{Vu}$ ), o que é comprovado pela diminuição acentuada dos valores de torque máximo, teor de gel e propriedades mecânicas.

Há ainda de se considerar nessa discussão o eventual efeito da degradação oxidativa da borracha natural ocasionada pela presença de elementos metálicos no resíduo RG. Investigações estão sendo conduzidas nesse sentido e, caso se mostre efetivo o efeito de degradação, o uso de antioxidantes mais específicos na formulação e/ou a remoção dos elementos mais nocivos do resíduo galvânico devem ser considerados.

\section{Conclusão}

A substituição parcial ou total do óxido de zinco, um conhecido ativador do processo de vulcanização de elastômeros, por um resíduo do processo de galvanoplastia denominado de RG foi investigada. Verificou-se que o resíduo apresenta um efeito catalítico sobre a vulcanização da borracha natural para o sistema testado. Há uma redução nos valores do tempo de pré-cura $\left(\mathrm{t}_{\mathrm{s1}}\right)$ e tempo ótimo de cura $\left(t_{90}\right)$. No entanto, quando RG está presente em quantidade 
equivalente ou superior ao $\mathrm{ZnO}$ nas composições, há um comprometimento das propriedades mecânicas.

Através da aplicação de um modelo cinético apropriado para o estudo da vulcanização de NR, observou-se que RG conduz a uma rápida formação de espécies químicas iniciais. Porém, tais espécies não preenchem os requisitos suficientes para dar seqüência de forma satisfatória a vulcanização. Fato que se reflete no baixo desempenho dos vulcanizados, em particular, os da composição M5 - somente RG como o ativador da mistura.

\section{Agradecimentos}

Os autores agradecem às agências de fomento CAPES, FAPERJ e CNPq pelo suporte financeiro e ao IMA / UFRJ pelo processamento e reômetro das misturas elastoméricas.

\section{Referências Bibliográficas}

1. Cioffi, R.; Lavorgna, M. \& Santoro, L. - Journal of Hazardous Materials, 89, p.165 (2002).

2. Silva, P. T. S.; Mello, N. T.; Duarte, M. M. M.; Conceição M.; Montenegro, B. S. M.; Araújo, A. N.; Neto, B. B. \& Silva, V. L. - Journal of Hazardous Materials, 128, p.39 (2006).

3. Suksabye, P.; Thiravetyan, P \& Nakbanpote, W. - Journal of Hazardous Materials, 160, p.56 (2008).

4. Coran, A. Y. - "Vulcanization", in: The Science and Technology of Rubber" - Mark, J. E., Erman, B., Eirich, F. R., (Eds.), Academic Press, New York (2005).

5. Morton, M. - "Rubber Technology", Kluwer Academic Publishers, Boston (1999).

6. da Costa, H. M.; Visconte, L. L. Y.; Nunes, R. C. R. \& Furtado, C. R. G. - Journal of Applied Polymer Science, 87, p.1194 (2003).
7. da Costa, H. M.; Visconte, L. L. Y.; Nunes, R. C. R. \& Furtado, C. R. G. - Journal of Applied Polymer Science, 87, p.1405 (2003).

8. da Costa, H. M.; Visconte, L. L. Y.; Nunes, R. C. R. \& Furtado, C. R. G. - Journal of Applied Polymer Science, 90, p.1519 (2003).

9. Russel, R. M.; Skinner, T. D. \& Watson, A. A. - Rubber Chemistry and Technology, 41, p.418 (1968).

10. Barbin, B. B. \& Rodgers, W.W. - "The Science of Rubber Compounding", in: The Science and Technology of Rubber, Mark, J. E., Erman, B., Eirich, F. R. (Eds.), Academic Press, New York (1994).

11. Vinod, V. S.; Varghese, S. \& Kuriakose, B. - Polymer Degradation and Stability, 75, p.405 (2002).

12. Lokander, M.; Reitberger, T. \& Stenberg, B. - Polymer Degradation and Stability, 75, p.405 (2002).

13. Coran, A. Y. - Rubber Chemistry and Technology, 37, p.689 (1964).

14. Coran, A. Y. - Rubber Chemistry and Technology, 68, p.351 (1995).

15. Cotten, R. G. - Rubber Chemistry and Technology, 45, p.129 (1972).

16. Ignatz-Hoover, F. \& To, B. H. - "Vulcanization", in: Rubber Compounding - Chemistry and Applications, Rodgers, B., (Ed.), Marcel Dekker, New York (2004).

17. Versloot, P.; Haasnoot, J. G.; Reedijk, J.; van Duin, M. \& Put, J. - Rubber Chemistry and Technology, 67, p.263 (1994)

18. Steudel, R.; Steudel, Y. \& Wong, M. W - Chemistry European Journal, 14, p.919 (2008).

Enviado: 16/04/09 Reenviado: 16/06/09

Aceito: 25/06/09 